\title{
MiR-193a-3p and miR-193a-5p suppress the metastasis of human osteosarcoma cells by down-regulating Rab27B and SRR, respectively
}

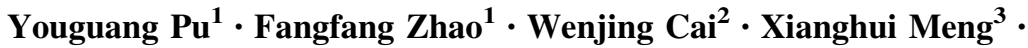 \\ Yinpeng $\mathrm{Li}^{4} \cdot$ Shanbao $\mathrm{Cai}^{1{ }^{1,3}}$
}

Received: 29 October 2015/Accepted: 17 February 2016/Published online: 25 February 2016

(c) The Author(s) 2016. This article is published with open access at Springerlink.com

\begin{abstract}
MicroRNAs have been identified as key players in the development and progression of osteosarcoma, which is the most common primary malignancy of bone. Sequencing-based miR-omic and quantitative real-time PCR analyses suggested that the expression of miR-193a$3 p$ and miR-193a-5p was decreased by DNA methylation at their promoter region in a highly metastatic osteosarcoma cell line (MG63.2) relative to their expression in the less metastatic MG63 cell line. Further wound-healing and invasion assays demonstrated that both miR-193a-3p and miR-193a-5p suppressed osteosarcoma cell migration and invasion. Moreover, introducing miR-193a-3p and miR193a-5p mimics into MG63.2 cells or antagomiRs into MG63 cells confirmed their critical roles in osteosarcoma metastasis. Additionally, bioinformatics prediction along with biochemical assay results clearly suggested that the secretory small GTPase Rab27B and serine racemase (SRR) were direct targets of miR-193a-3p and miR-193a$5 \mathrm{p}$, respectively. These two targets are indeed involved in the miR-193a-3p- and miR-193a-5p-induced suppression of osteosarcoma cell migration and invasion. MiR-193a-3p
\end{abstract}

Youguang Pu and Fangfang Zhao have contributed equally to this work.

Shanbao Cai

shanbaocai651116@163.com

1 Cancer Epigenetics Program, Anhui Cancer Hospital, Hefei 230031, Anhui, China

2 Indiana University School of Medicine, Indianapolis, IN 46202, USA

3 Department of Orthopedic Surgery, Anhui Cancer Hospital, Hefei 230031, Anhui, China

4 Xinxiang Medical University, Xinxiang 453000, Henan, China and miR-193a-5p play important roles in osteosarcoma metastasis through down-regulation of the Rab27B and SRR genes and therefore may serve as useful biomarkers for the diagnosis of osteosarcoma and as potential candidates for the treatment of metastatic osteosarcoma.

Keywords Rab27B $\cdot$ SRR $\cdot$ MiR-193a-3p and miR-193a$5 \mathrm{p} \cdot$ Osteosarcoma $\cdot$ Metastasis

\begin{tabular}{ll}
\multicolumn{2}{l}{ Abbreviations } \\
OS & Osteosarcoma \\
MiR & MicroRNA \\
UTR & Untranslated region \\
Rab27B & Member RAS oncogene family \\
SRR & Serine racemase \\
3PM/5PM & MiR-193a-3p and miR-193a-5p mimic \\
3PA/5PA & MiR-193a-3p and miR-193a-5p \\
BSP & Bisulfite sequencing PCR
\end{tabular}

\section{Introduction}

Osteosarcoma is the most common primary bone malignancy in children and young adults [1,2]. Approximately $80 \%$ of osteosarcoma patients have metastatic disease at the time of diagnosis, and metastasis is a consistent problem in tumor prognosis and treatment [3]. The molecular mechanisms that lead to the development and metastasis of osteosarcoma are poorly understood [4-6]. Further exploration of this area will help in the development of effective strategies in the diagnosis, treatment and prognosis of osteosarcoma. Previous studies have established a highly tumorigenic and metastatic human osteosarcoma cell line, 
MG63.2, derived from the less metastatic parental MG63 cell line [7]. The MG63.2 cell line facilitates the exploration of crucial players in osteosarcoma metastasis [8].

MicroRNAs (miRNAs), a class of small non-coding RNA molecules, play critical roles in a variety of biological events, including development, cell proliferation and cell differentiation [9-11]. MiRNAs negatively regulate gene expression by binding to the $3^{\prime}$-untranslated regions (UTRs) of the corresponding target mRNAs of proteincoding genes, thereby leading to mRNA degradation or translation inhibition [12-15]. Multiple miRNAs are involved in the invasion and metastasis of different types of cancers, including gastric cancer, breast cancer, hepatocellular carcinoma and colorectalcancer [16-19]. In osteosarcoma, a few miRNAs have been reported to regulate metastasis. For instance, miR-16 inhibits cell proliferation by targeting IGF1R and the Raf1-MEK1/2-ERK1/2 pathway in osteosarcoma [20], and miR-802 promotes osteosarcoma cell proliferation by down-regulating the p27 cell-cycle inhibitor [21]. Moreover, miR-212 inhibits osteosarcoma cell proliferation and invasion by downregulating Sox4 [22]. However, the prospects of these miRNAs in the clinical treatment of osteosarcoma have not been evaluated. Novel miRNAs that may potentially serve as new therapeutic targets or biomarkers must urgently be identified.

In the present study, we aimed to identify novel miRNAs associated with osteosarcoma metastasis. From a sequencing-based miR-omic study and referred to the relevant literatures, we found two miRNAs, miR-193a-3p and miR-193a-5p, that play a role in osteosarcoma metastasis. These two miRNAs, which are derived from the same precursor, have different sequences and therefore target different mRNAs [23]. These miRNAs have been implicated in the tumorigenesis and progression of several types of cancer [24-28]. In addition, we identified the target genes of miR-193a-3p and miR-193a-5p, and we proposed the potential pathway that might be associated with osteosarcoma metastasis. Therefore, miR-193a-3p and miR-193a-5p, as well as their targets, might serve as useful biomarkers for the diagnosis of osteosarcoma and as potential candidates for the treatment of metastatic osteosarcoma.

\section{Materials and methods}

\section{Cell lines and culture}

The MG63 osteosarcoma cell line was purchased from the American Type Culture Collection (ATCC; NO. CRL1427), and the MG63.2 cell line was kindly provided by Dr. Luu from the University of Chicago [7]. The cells were cultured in Dulbecco's modified Eagle's medium (DMEM; Invitrogen, Carlsbad, CA, USA) supplemented with $10 \%$ fetal bovine serum (FBS; Invitrogen) and $1 \%$ glutamine at $37{ }^{\circ} \mathrm{C}$ in $5 \% \mathrm{CO}_{2}$.

\section{RNA-seq analysis}

RNA-seq analysis was performed by BGI-Tech of China, and RNA-seq library preparation and sequencing were performed by BGI (Shenzhen, China). Following purification, RNA was fragmented using divalent cations at an elevated temperature, and first-strand cDNA was synthesized using random hexamer primers and Superscript TMIII (Invitrogen ${ }^{\mathrm{TM}}$, Carlsbad, CA, USA). Second-strand cDNA was synthesized using buffer, dNTPs, RNaseH, and DNA polymerase I. Short fragments were purified with a QiaQuick PCR extraction kit (Qiagen) and resolved with EB buffer for end reparation and poly (A) addition. The short fragments were subsequently connected using sequencing adapters. After agarose gel electrophoresis, suitable fragments were used as templates for PCR amplification. During the QC steps, an Agilent 2100 Bioanaylzer and an ABI StepOnePlus Real-Time PCR System were used in quantification and qualification of the sample library. Finally, the library (200 bp insert) was sequenced using Illumina HiSeq 2000 (Illumina Inc., San Diego, CA, USA). The single-end library was prepared following the protocol of the IlluminaTruSeq RNA Sample Preparation Kit (Illumina).

\section{Transfection of mimics, antagomiRs, siRNAs and overexpression plasmids}

The mimics, antagomiRs, siRNAs, scramble sequence negative control (NC) and riboFECT CP transfection kits were supplied by Guangzhou Ribobio (Guangzhou, China). The mammalian expression constructs for GFP-tagged Rab27B (EX-Q0376-M98-5) and serine racemase (SRR) (EX-W0524-M98-5) were supplied by Guangzhou Fulengen (Guangzhou, China). Transfection of the ribonucleic acid reagents or plasmids mentioned in this paper as well as the reporter plasmids in the Cignal Finder ${ }^{\mathrm{TM}}$ Pathway Reporter Arrays (SABiosciences, USA) was performed according to the manufacturer's instructions. The following sequences were used in this study:

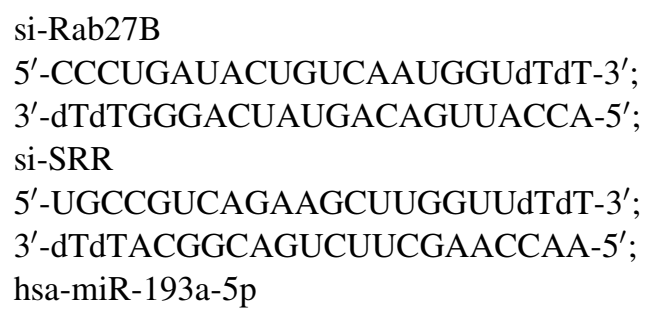


antagomiR: 5'-UCAUCUCGCCCGCAAAGACCCA-3'; mimics:

sense, $5^{\prime}$-UGGGUCUUUGCGGGCGAGAUGA- ${ }^{\prime}$ antisense, 5'-UCAUCUCGCCCGCAAAGACCCA-3'; hsa-miR-193a-3p

antagomiR: 5 -ACUGGGACUUUGUAGGCCAGUU$3^{\prime}$;

mimics:

sense, 5'-AACUGGCCUACAAAGUCCCAGU- ${ }^{\prime}$ antisense, $5^{\prime}$-ACUGGGACUUUGUAGGCCAGUU- $3^{\prime}$.

Mimics were composed of double-stranded RNA with no chemical modifications. The $3^{\prime}$ ends of the antagomiR and agomiR oligo nucleotides were conjugated to cholesterol, and all the bases were $2^{\prime}-\mathrm{O}$ methylated $(\mathrm{OMe})$. All of the oligo nucleotides were deprotected, desalted and purified by high-performance liquid chromatography.

\section{Luciferase reporter assay}

The full-length $3^{\prime}$-UTR of human SRR (1386 bp) and a partial 3'-UTR of Rab27B (1034 bp; 830-1863 from a $6103 \mathrm{bp}$ full-length sequence) containing miR-193a-5pand miR-193a-3p-binding sites, respectively, were cloned downstream of the firefly luciferase gene in pGL3 (Invitrogen) to construct pGL3-luc-Rab27B and pGL3-luc-SRR, respectively. All of the constructs were confirmed by restriction digestion. Cells were seeded into 96-well plates at approximately $1 \times 10^{4}$ cells per well and transfected with a mixture of $50 \mathrm{ng}$ of pGL3-luc-Rab27B or pGL3-lucSRR, $5 \mathrm{ng}$ of Renilla luciferase and 5 pmol mimics or NC nucleotides using the riboFECT $\mathrm{CP}$ transfection reagents according to the manufacturer's instructions. Both firefly and Renilla luciferase activities were measured $24 \mathrm{~h}$ after transfection using a Dual-Luciferase Reporter Assay System (Promega) on the Promega GloMax 20/20 luminometer. The relative firefly luciferase activities were normalized with the Renilla luciferase activities as a control for transfection efficiency.

\section{Pathway luciferase reporter constructs}

The NC construct, which encodes firefly luciferase under the control of a basal promoter element (TATA box) without any additional transcriptional response elements, is critical for identifying specific effects and determining background reporter activity. The reporter construct encodes the firefly luciferase reporter gene under the control of a basal promoter element (TATA box) joined to tandem repeats of a specific transcriptional response element. The positive control construct is a mix of constructs that constitutively express GFP, firefly luciferase and Renilla luciferase. The analysis was performed according to the manufacturer's instruction (SABiosciences). Briefly, the cells were triple transfected with each firefly luciferase reporter construct in combination with the Renilla luciferase construct using the riboFECT $\mathrm{CP}$ transfection reagent, and both luciferase activities in cell extracts at $24 \mathrm{~h}$ after transfection were measured using the Promega Dual-Luciferase Reporter assay on the PromegaGloMax 20/20 luminometer. Firefly luciferase activities from each set were normalized to the Renilla luciferase activity to control for inter-transfection bias. The relative luciferase activities (luciferase unit) of the pathway reporter over the negative control in the transfected cells were calculated as a measure of the pathway activity.

\section{RNA analysis}

Total RNA was isolated using the TRIzol reagent (Tiangen Biotech Co., Ltd., Beijing, China). For mRNA analysis, cDNA was synthesized from total RNA by oligo-dT priming by using a primeScript RT reagent kit (Tiangen Biotech Co., Ltd.). The Rab27B and SRR mRNA levels were measured by quantitative real-time polymerase chain reaction (qRTPCR) using gene-specific fluorescent TaqMan probes together with $\beta$-actin using a different fluorescent probe (provided by ShingGene, Shanghai, China) on an FTC-3000P PCR instrument (FUNGLYN BIOTECH INC, Toronto, ON, Canada). For miRNA analysis, cDNA was synthesized with specific stem-loop primers and quantified by using a SYBR Green-based RT-PCR assay, and normalization to the U6 reads for miRNA or to $\beta$-actin for mRNA was performed using the $2^{-\Delta \Delta C_{\mathrm{t}}}$ method before calculating the relative mRNA levels between MG63 and MG63.2 cells. The following primers and probes for complementary DNA synthesis and qRT-PCR analysis were used:

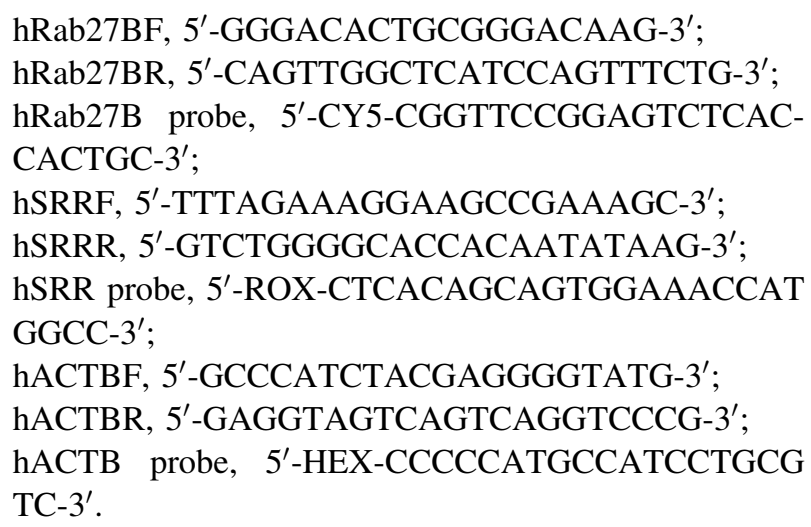

\section{Western blotting analysis}

Cell lysates in $1 \times$ SDS loading buffer $(60 \mathrm{mM}$ Tris-HCl, pH6.8; $2 \%$ SDS; $20 \%$ glycerol; $0.25 \%$ bromophenol 
blue; and 1.25\%2-mercaptoethanol) were incubated at $100{ }^{\circ} \mathrm{C}$ for $10 \mathrm{~min}$ to facilitate sample loading for conventional western blotting analysis. Anti-Rab27B (134121-AP), anti-SRR (17955-1-AP) and anti-GAPDH (600041-lg) antibodies were provided by San Ying Biotechnology, China. The target proteins were then probed with an anti-rabbit IgG peroxidase-conjugated antibody (LP1001b) (San Ying Biotechnology, China) and detected by an enhanced chemiluminescence reaction (ThermoFisher Scientific, Waltham, MA, USA). The relative protein levels were quantified using densitometry with a Gel-Pro Analyzer (Media Cybernetics, Rockville, MD, USA). The intensities of the target bands relative to GAPDH were quantified by densitometry, and these values are indicated under each band.

\section{Bisulfite sequencing PCR (BSP) analysis}

Genomic DNA was isolated using a standard phenol/ chloroform purification method, qualified via agarose gel electrophoresis, and treated with an ammonium bisulfatebased bisulfite conversion method [29, 30]. The PCR fragments from the converted DNA were sequenced and analyzed. Raw sequence data files were processed, and the area ratio $(\%)$ of $\mathrm{C}$ to $\mathrm{C}+\mathrm{T}$ of the primary $\mathrm{CpG}$ dinucleotide was calculated as the percentage of methylation and was then plotted.

\section{Wound-healing assays}

For cell motility assays, cells stably expressing mimics, antagomiRs or NC were seeded in 24-well plates and cultured to near confluence. After $6 \mathrm{~h}$ of culture in DMEM without FBS, a linear wound was carefully made using a sterile $10 \mu$ pipette tip across the confluent cell monolayer, and the cell debris was removed by washing with phosphate-buffered saline. The cells were incubated in DMEM plus $10 \% \mathrm{FBS}$, and the wounded monolayers were then photographed at $0,8,24$ and $48 \mathrm{~h}$ after wounding.

\section{In vitro invasion assays}

Cell invasion assays were performed in a 24-well plate with $8 \mathrm{~mm}$ pore size chamber inserts (Corning, New York, NY, USA). For invasion assays, $1 \times 10^{4}$ cells stably expressing mimics, antagomiRs or NC were placed into the upper chamber in each well with the matrigel-coated membrane, which was diluted in serum-free culture medium. In the assay, cells were suspended in $100 \mu \mathrm{l}$ of DMEM without FBS when they were seeded into the upper chamber. In the lower chamber, $500 \mu$ l of DMEM supplemented with $10 \%$ FBS was added. After incubation for $30 \mathrm{~h}$ at $37{ }^{\circ} \mathrm{C}$ and $5 \% \mathrm{CO}_{2}$, the membrane inserts were removed from the plate, and non-invading cells were removed from the upper surface of the membrane. Cells that moved to the bottom surface of the chamber were stained with $0.1 \%$ crystal violet for $30 \mathrm{~min}$. The cells were then imaged and counted in at least five random fields using a CKX41 inverted microscope (Olympus, Tokyo, Japan). The assays were conducted three independent times.

\section{Bioinformatics analysis}

RAB27B, SRR and key pathway genes (TGF $\beta$ : TGFB1; MYC/Max: MYC; and ATF2/ATF3/ATF4: ATF2, ATF3, and ATF4) were used as query genes to predict potential interactions in the GeneMANIA database (http://genema nia.org/). GeneMANIA uses a fast heuristic algorithm to integrate functional association networks containing all input genes. To simplify the model, the shortest paths between query genes were extracted from the networks. Those interactions reported in the literature were chosen first to obtain the true functional role of each interaction in the connected network of all query genes.

\section{Statistical analysis}

Data are presented as the means, and error bars indicate the standard deviation (SD). All statistical analyses were performed with Excel (Microsoft, Redmond, WA, USA). Two-tailed Student's $t$ test, one-way analysis of variance (ANOVA) or the Mann-Whitney $U$ test was used to calculate statistical significance. A $P$ value of $<0.05$ was considered to be significant.

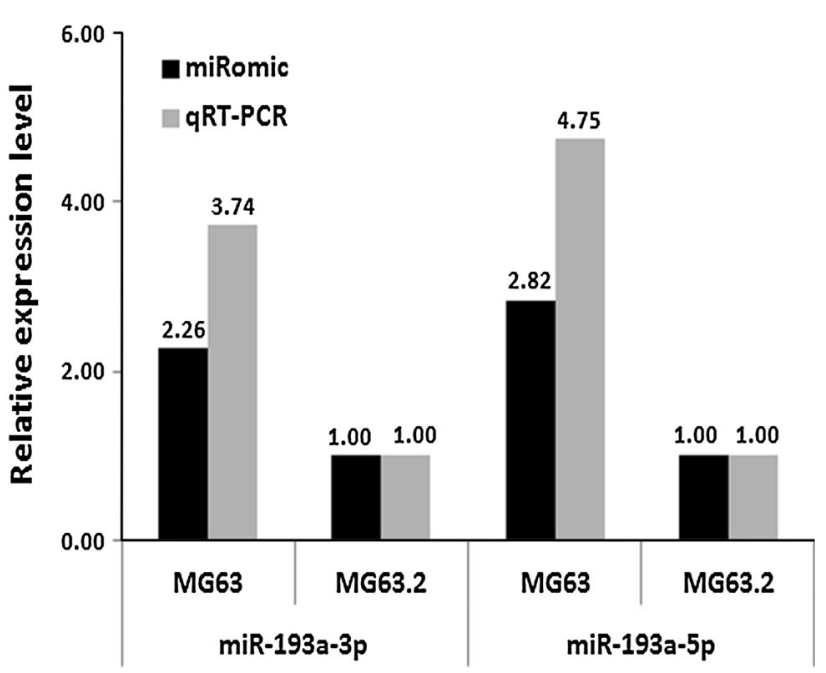

Fig. 1 The relative miR-193a-3p and miR-193a-5p expression levels (fold change) in the MG63 and MG63.2 cell lines as measured by miR-omic and qRT-PCR analyses are shown in the plot 
A

mir-193a bspf

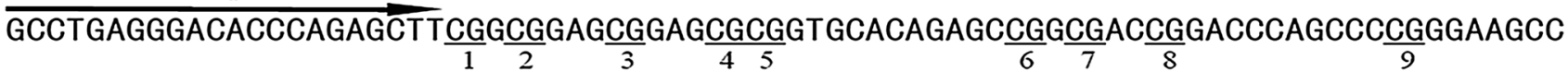

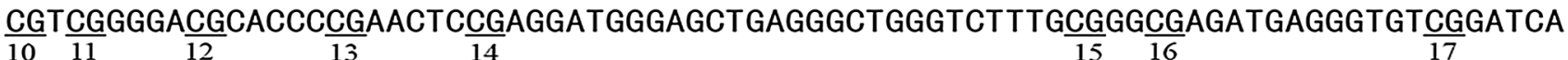

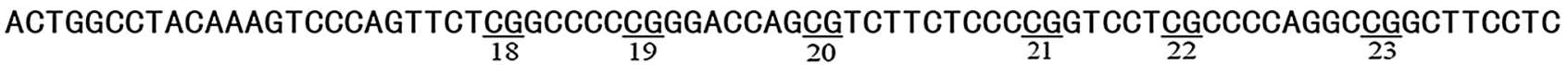
CCGGGCTGGCGTGCGCTCCGGGCCAGGCTGCCTCTCAGGT mir-193a bspt

B

MG63.2

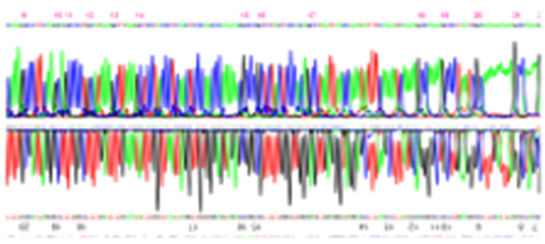

Forward

Reverse

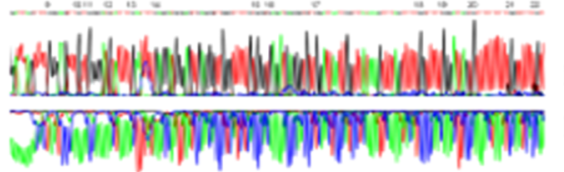

Forward

Reverse
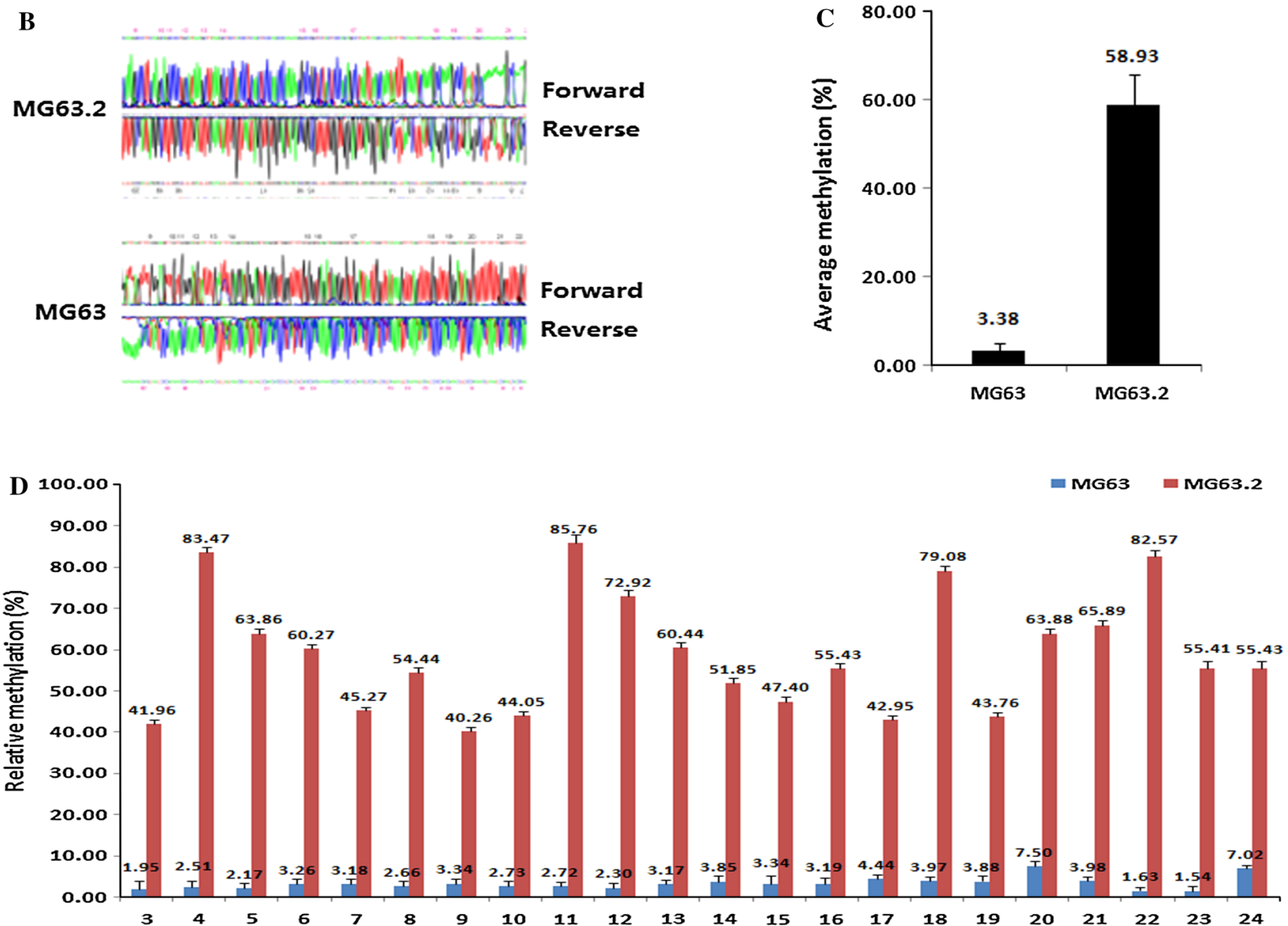

Fig. 2 Differential methylation of the miR-193a gene in MG63 versus MG63.2 cells. The $\mathrm{CpG}$ dinucleotides and the BSP primers of the miR-193a gene are shown (a). The original sequencing results of

\section{Results}

MiR-193a-3p and miR-193a-5p are hypermethylated and down-regulated in the highly metastatic MG63.2 osteosarcoma cell line

MiR-193a-3p and miR-193a-5p have been characterized as tumor suppressors in several types of cancer such as nonsmall cell lung cancer (NSCLC) [24, 28], prostate cancer the bisulfite-converted DNA are shown (b). The percentage of $\mathrm{CpG}$ methylation is summarized in the plot $(\mathbf{c}, \mathbf{d})$

[21], breast cancer [31], head and neck squamous cell carcinomas [32], and colorectal cancer [33]. However, the roles of miR-193a-3p and miR-193a-5p in osteosarcoma cells remain unclear. According to a sequencing-based miR-omic study in two human osteosarcoma cell lines (weakly metastatic MG63 cells and highly metastatic MG63.2 cells), both miR-193a-3p and miR-193a-5p were found to be among the top differentially expressed miRs. Quantitative real-time polymerase chain reaction (qRT- 
A

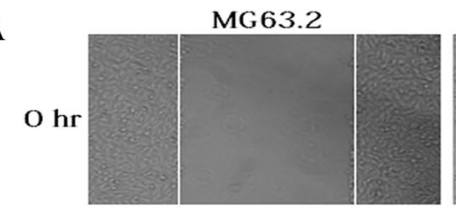

$48 \mathrm{hr}$
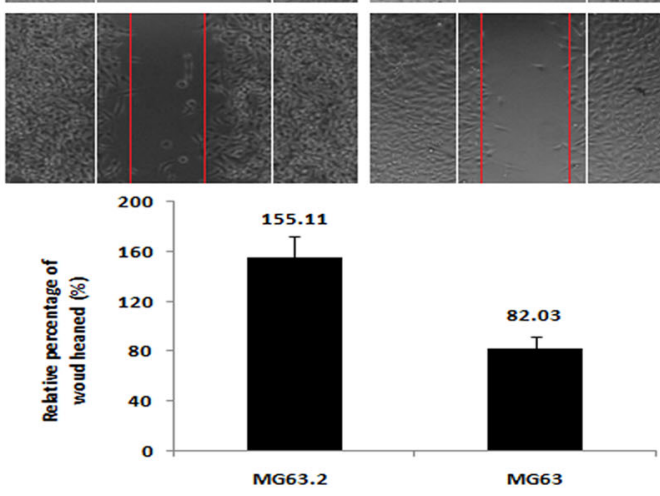

C
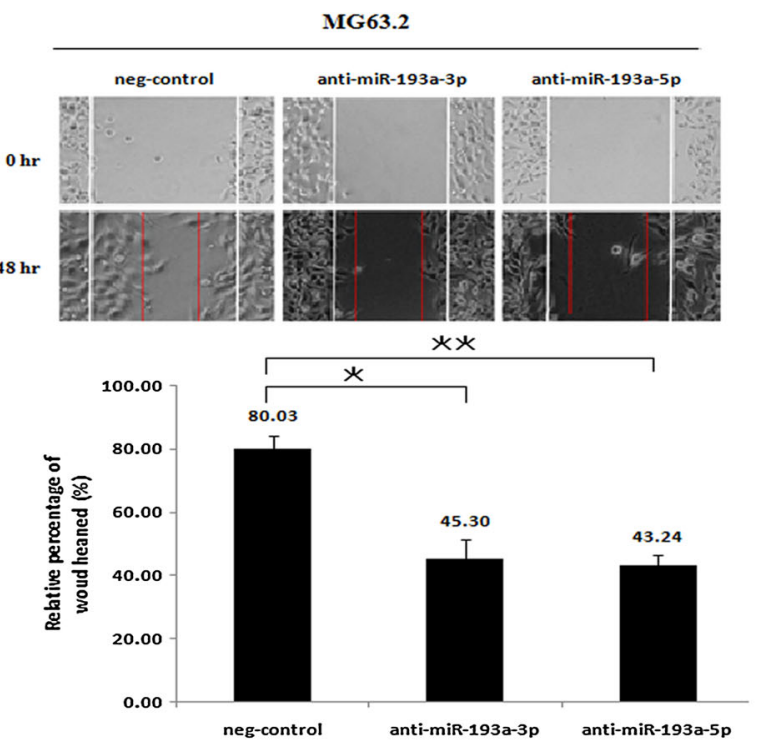

$\mathbf{E}$
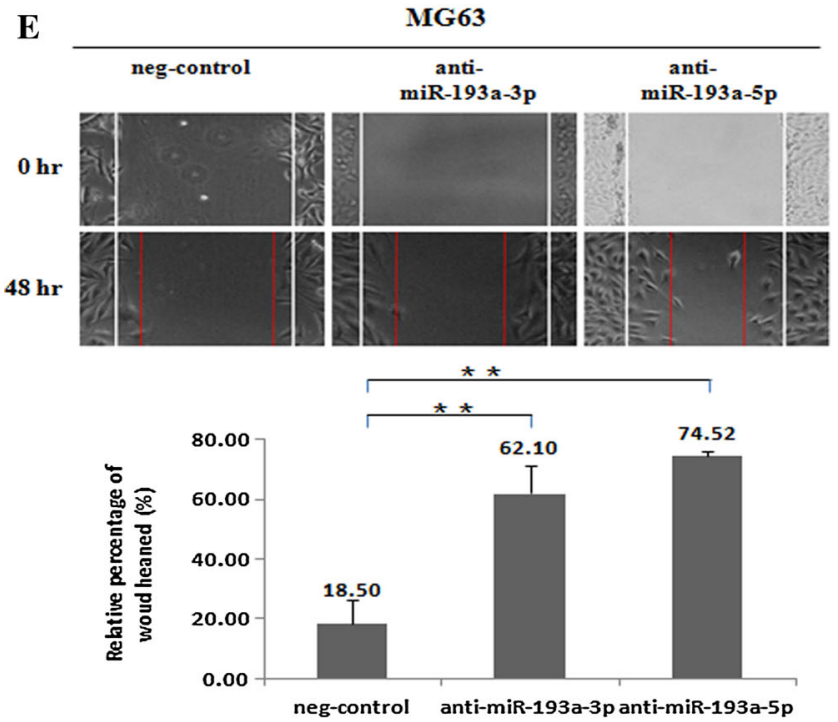

B

MG63

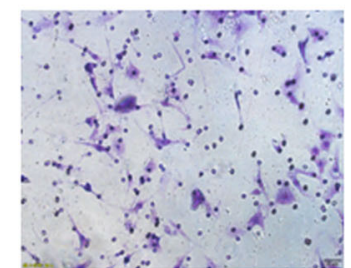

MG63.2
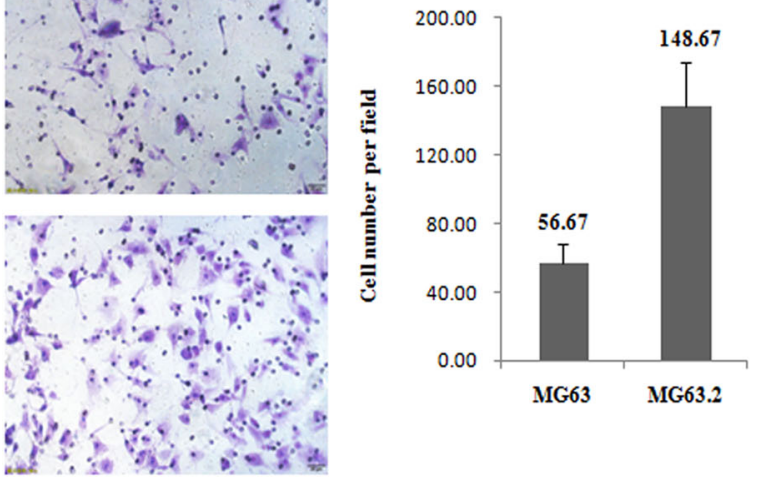

D
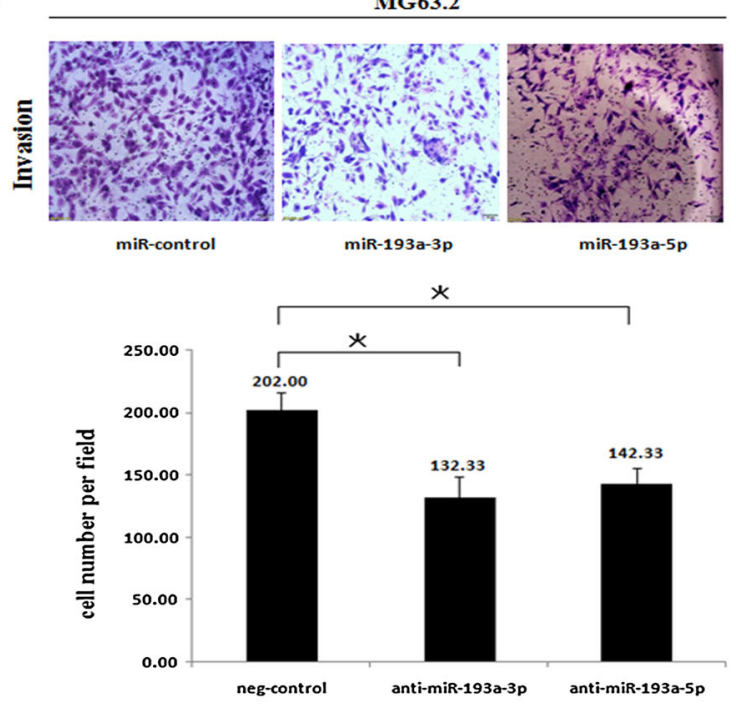

F
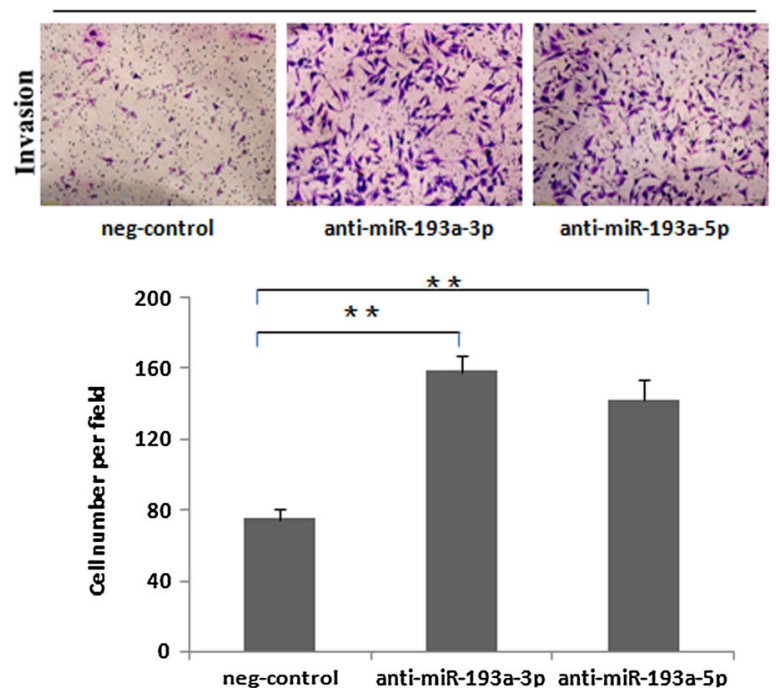
4Fig. 3 MiR-193a-3p and miR-193a-5p expression levels are reduced in highly metastatic cancer cells, consequently affecting cell migration and invasion.MG63.2 cells showed a higher capacity for migration and invasion than MG63 cells (a, b). Wound-healing assays that determine the migration and invasion of MG63 and MG63.2 cells were performed with transient expression of the miR193a-3p mimic (3PM), miR-193a-5p (5PM) mimic, or negative control (NC) in MG63.2 cells as well as with the miR-193a-3p antagomiR (3PA), miR-193a-5p antagomiR (5PA) or NC (in MG63 cells) (c, d). Invasion assays for MG63.2 and MG63 cells were conducted after transduction with the same set of constructs $(\mathbf{e}, \mathbf{f})$. The data are representative of three independent experiments. $* P<0.05$; $* * P<0.01$ by Student's $t$ test

PCR) analyses verified that miR-193a-3p and miR-193a-5p expression was lower in the highly metastatic MG63.2 cells than in the weakly metastatic MG63 cells (the expression ratios of miR-193a-3p and miR-193a-5p in MG63.2 with MG63 were 1.00:3.74 and 1.00:4.75, respectively; Fig. 1). To further investigate the underlying mechanism of decreased miR-193a-3p and miR-193a-5p expression in highly metastatic MG63.2 cells, the methylation status of the miR-193a promoter regions in both MG63 and MG63.2 cells were assessed using BSP. Of the total $27 \mathrm{CpG}$ sites, 22 were methylated at varying ratios (Fig. 2). The average methylation ratio of the miR-193a gene in MG63.2 cells was approximately 20-fold higher than that in MG63 cells (58.93 vs. 3.38; Fig. 2c). Thus, increased methylation was negatively correlated with the expression of both miR193a-3p and miR-193a-5p.

\section{MiR-193a-3p and miR-193a-5p suppress osteosarcoma cell migration and invasion in vitro}

The differential methylation status and expression of miR193a-3p and miR-193a-5p between MG63 and MG63.2 cells indicate their potential roles in the metastasis of osteosarcoma. Therefore, we compared the migration and invasion capabilities of MG63.2 and MG63 cells using wound-healing and Matrigel invasion assays, respectively. As previously reported, MG63.2 cells showed a higher capacity for migration and invasion than MG63 cells (Fig. 3a, b), suggesting the active metastatic ability of MG63.2 cells. Loss- and gain-of-function studies of miR$193 a-3 p$ and miR-193a-5p were conducted by transiently transfecting miR-193a-3p and miR-193a-5p mimics or antagomiRs into MG63.2 and MG63 cells. As shown in Fig. 3c, d, both the migration and invasion of miR-193a-3p and miR-193a-5p mimic-transfected MG63.2 cells were significantly suppressed compared with those of control cells. In contrast, the migration and invasion of MG63 cells were increased by transfection of miR-193a-3p and miR193a-5p antagomiRs (Fig. 3e, f).
Rab27B and SRR are direct targets of miR-193a-3p and miR-193a-5p

To further clarify the underlying molecular mechanisms of the suppressive effects of miR-193a-3p and miR-193a$5 \mathrm{p}$ on osteosarcoma cell metastasis, the predicted target genes of miR-193a-3p and miR-193a-5p were selected by using three commonly used miR-target-predicting methods as follows: miRDB, miRBase, and TargetScan (Fig. 4a). RNA-Seq data of MG63 and MG63.2 cells were then used to screen the possible candidates predicted to be expressed with the opposite trend to that of miR-193a-3p and miR-193a-5p between MG63 and MG63.2 cells (Fig. 4b, c). Subsequent qRT-PCR assays verified that the mRNA expression of Rab27B (target of miR-193a-3p) and SRR (target of miR-193a-5p), both from the candidate list, was significantly higher in MG63.2 cells than in MG63 cells (Fig. 4b, c). Western blot analysis also suggested that Rab27B and SRR protein levels were relatively higher in MG63.2 cells than that in MG63 cells (Fig. 4d). All of these results correlated well with the negative regulation of the target genes by miR-193a-3p and miR-193a-5p.

We next tested Rab27B and SRR expression with the addition of miR-193a-3p and miR-193a-5p mimics (termed 3PM and 5PM, respectively) in MG63.2 cells or miR-193a$3 p$ and miR-193a-5p antagomiRs (termed 3PA and 5PA, respectively) in MG63 cells. Both mRNA and protein levels of Rab27B were suppressed by 3PM in MG63.2 cells and increased by 3PA in MG63 cells compared with those in control cells (Fig. 4e-g). Similarly, the mRNA and protein levels of SRR were suppressed by 5PM in MG63.2 cells and increased by 5PA in MG63 cells compared with those in control cells (Fig. 4e-g). These results suggested that Rab27B is the target of miR-193a-3p and that SRR is the target of miR-193a-5p.

To further determine if Rab27B and SRR are the direct targets of miR-193a-3p and miR-193a-5p, respectively, we searched for the miR-193a-3p- and miR-193a-5p-binding sites in the $3^{\prime}$-UTRs of Rab27B and SRR and generated pGL3-Rab27B UTR wild type (WT) and pGL3-SRR UTR WT constructs (Fig. 4h). Both constructs and the pGL3control were transfected into MG63 and MG63.2 cells to determine if the differential expression of miR-193a-3p and miR-193a-5p was functional in the context of the two cell lines. Both UTR-containing constructs showed comparable luciferase activities in MG63.2 and MG63 cells (Fig. 4i). The transfection of 3PM and 5PM in MG63.2 cells significantly decreased the luciferase activity of the Rab27B-UTR and SRR-UTR constructs, respectively, compared with that in control cells (Fig. 4j). In contrast, 
A

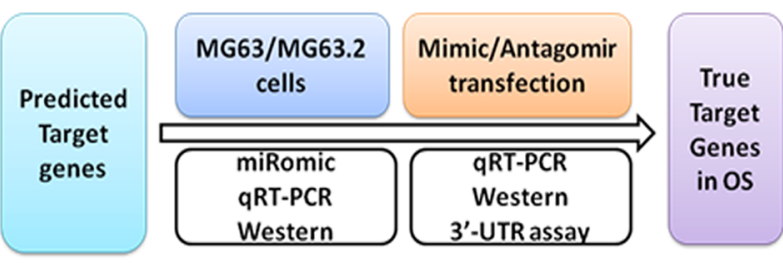

C

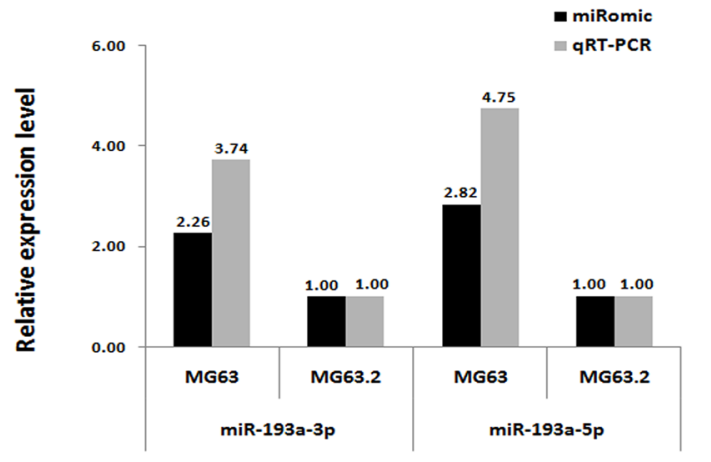

E
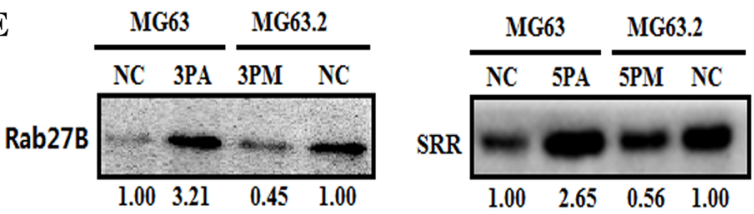

GAPDH

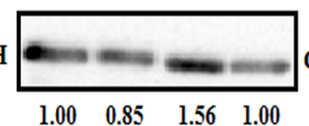

GAPDH

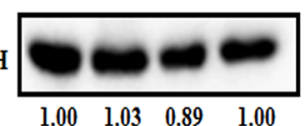

G

\begin{tabular}{c|c|c|c|} 
Genes & Cell line & 3p or 5p & NC \\
\hline \multirow{2}{*}{ Rab27B } & MG63 & 0.09 & 1.00 \\
\cline { 2 - 4 } & MG63.2 & 30.21 & 1.00 \\
\hline \multirow{2}{*}{ SRR } & MG63 & 0.21 & 1.00 \\
\cline { 2 - 4 } & MG63.2 & 5.36 & 1.00 \\
\hline
\end{tabular}

B

\begin{tabular}{c|c|c|c|}
\multirow{2}{*}{ Genes } & Cell line & RNA-seq & qRT-PCR \\
\hline \multirow{2}{*}{ Rab27B } & MG63 & 1.00 & 1.00 \\
\cline { 2 - 4 } & MG63.2 & 7.10 & 13.25 \\
\hline \multirow{2}{*}{ SRR } & MG63 & 1.00 & 1.00 \\
\cline { 2 - 4 } & MG63.2 & 4.12 & 4.37 \\
\hline
\end{tabular}

D

MG63 MG63.2

MG63 MG63.2

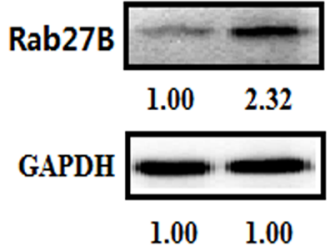

SRR

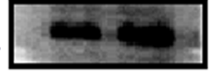

\begin{tabular}{ll}
$1.00 \quad 2.05$ \\
\hline
\end{tabular}

GAPDH

$\begin{array}{ll}1.00 & 1.34\end{array}$

F

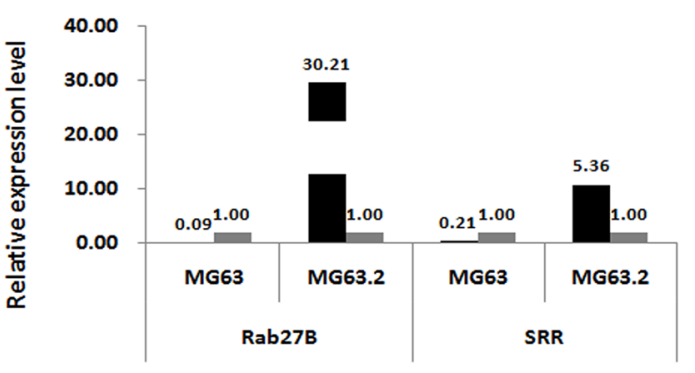

H
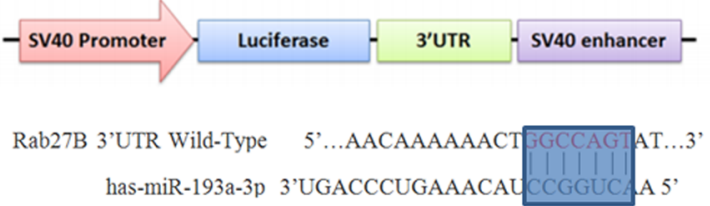

SRR

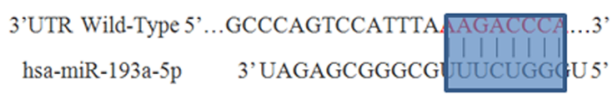

I

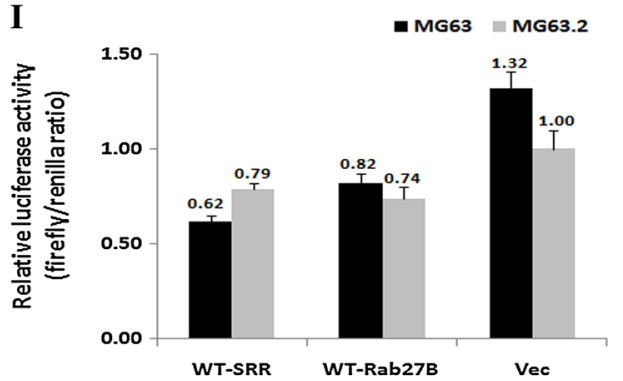

Fig. 4 MiR-193a-3p and miR-193a-5p target gene expression is negatively correlated with miR-193a-3p and miR-193a-5p expression. a The experimental scheme. SRR and Rab27B gene expression in MG63 versus MG63.2 cells at the mRNA level based on RNA-Seq analysis (b) and qRT-PCR (c). SRR and Rab27B expression in MG63 versus MG63.2 cells at the protein level (d). SRR and Rab27B gene expression in miR-193a-3p or miR-193a-5p mimic (3PM/5PM)transfected MG63.2 cells and miR-193a-3p or miR-193a-5p antagomiR (3PA/5PA)-transfected MG63 cells at the protein level as assessed by western blotting analysis (e) and at the mRNA level as
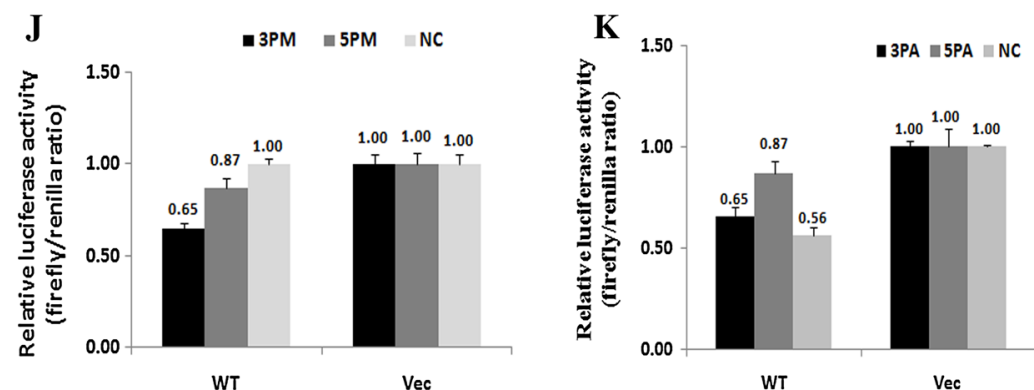

assessed by qRT-PCR (f, $\mathbf{g})$. h A schematic map of the pGL3-based luciferase reporter constructs where the UTR region ( $3^{\prime}$-UTR) of SRR or Rab27B gene was incorporated downstream of the luciferase gene. The relative luciferase activity (fold) of pGL3 with the Rab27B-UTR or SRR-UTR sequence relative to the control vector (VEC, with no UTR sequence) was determined in cells transfected with the miR193a-3p mimic (3PM), antagomiR (3PA) or scramble negative control (NC) (i-k). Representative results from three independent experiments are shown. ${ }^{*} P<0.05 ; * * P<0.01$ by Student's $t$ test 


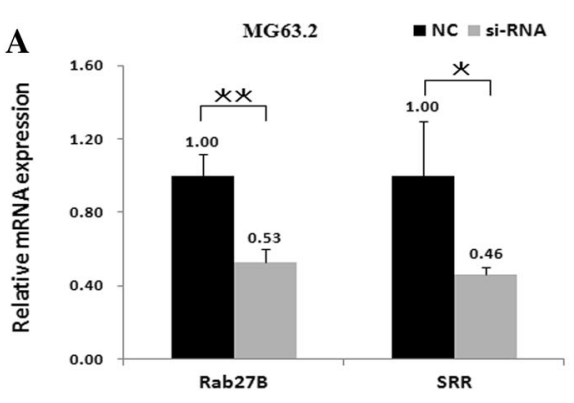

B

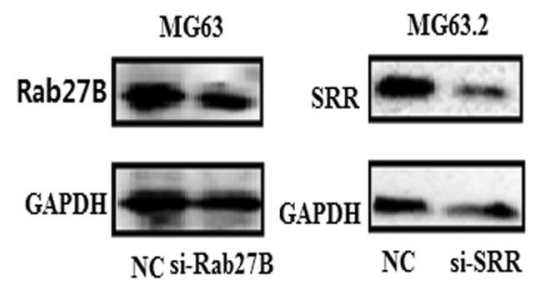

C

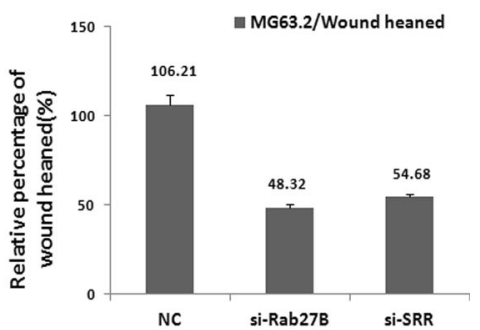

\section{$\mathbf{E}$}

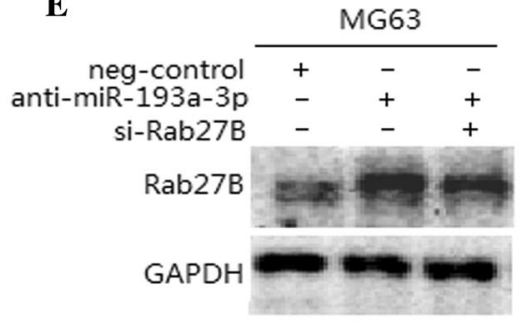

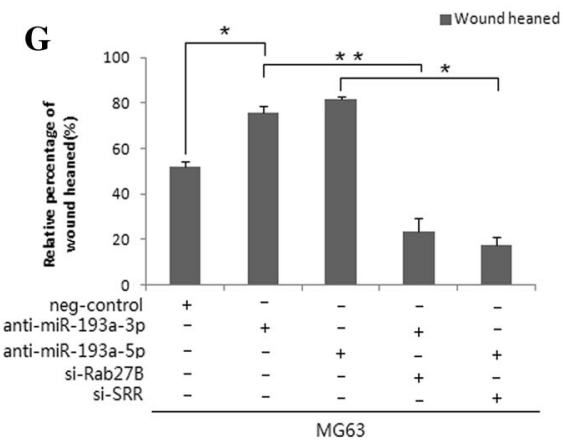

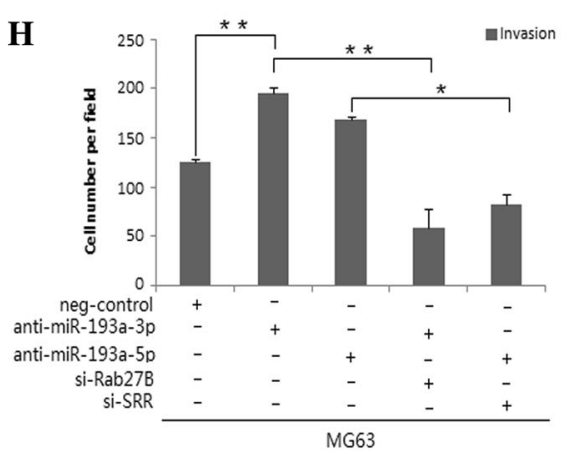

I GFP GFP-SRR

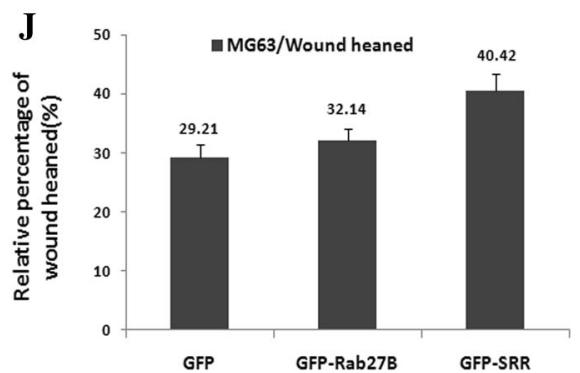

$\mathbf{K}$

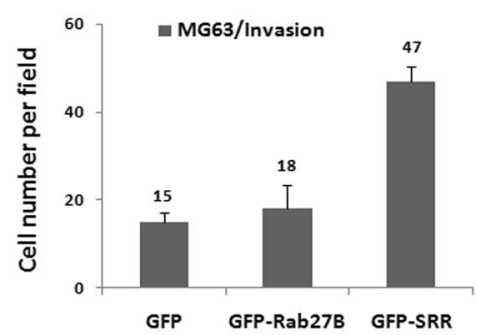

$\mathbf{L}$

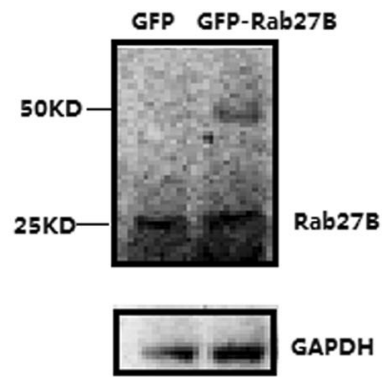

Fig. 5 Rab27B and SRR are involved in the miR-193a-3p- and miR193a-5p-induced suppression of osteosarcoma cell migration and invasion. Real-time PCR and western blot analyses of Rab27B and SRR expression in MG63.2 cells transfected with si-Rab27B, si-SRR or the negative control (NC). GAPDH was used as an internal control (a, b). Wound-healing and invasion assays were performed in MG63.2 cells after transfection with NC, si-Rab27B, si-SRR (c, d). Western blot analysis of Rab27B and SRR in MG63 cells stably expressing miR-193a-3p or miR-193a-5p antagomiR after transfection with si-Rab27B, si-SRR or NC. GAPDH was used as an internal

the transfection of 3PA and 5PA in MG63 cells increased the luciferase activities of the Rab27B-UTR and SRR-UTR constructs, respectively, compared with the level in control control (e, f). Wound-healing and invasion assays using MG63 cells stably expressing miR-193a-3p or miR-193a-5p antagomiR after transfection with si-Rab27B, si-SRR or NC $(\mathbf{g}, \mathbf{h})$. The data are representative of three independent experiments. Western blot analysis of Rab27B and SRR in MG63 cells stably overexpressing GFP-tagged Rab27B and SRR proteins or GFP alone. GAPDH was used as an internal control (i, j). Wound-healing and invasion assays using MG63 cells stably overexpressing GFP-tagged Rab27B and SRR proteins or GFP $(\mathbf{k}, \mathbf{l})$. The data are representative of three independent experiments. $* P<0.05 ; * * P<0.01$ by Student's $t$ test

cells (Fig. 4k). Thus, miR-193a-3p and miR-193a-5p decrease the expression of Rab27B and SRR, respectively, by directly targeting their $3^{\prime}$-UTRs. 


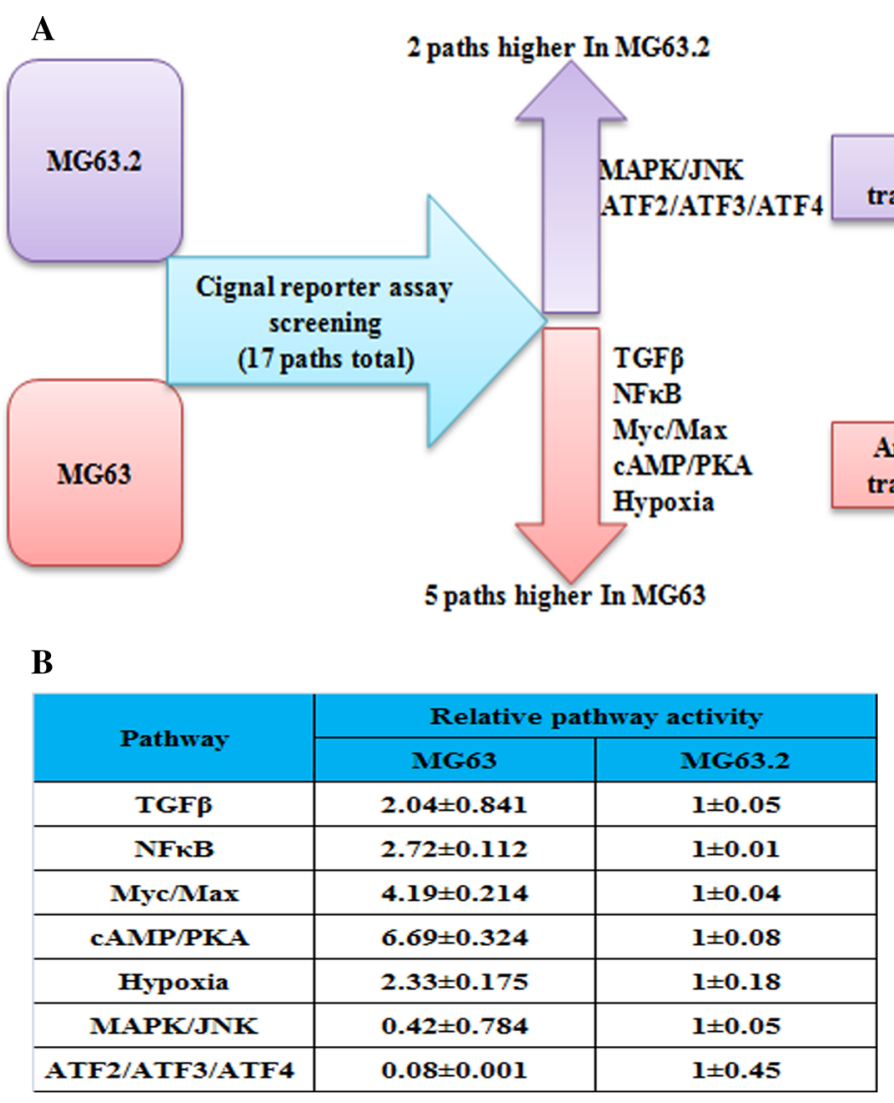

D

\begin{tabular}{c|c|c|c|} 
Pathway & NC & si-Rab27B & si-SRR \\
\hline TGFB & $1 \pm 0.04$ & $2.17 \pm 0.11$ & $4.12 \pm 0.31$ \\
\hline NFKB & $1 \pm 0.14$ & $0.73 \pm 0.45$ & $1.34 \pm 0.45$ \\
\hline Myc/Max & $1 \pm 0.01$ & $8.45 \pm 0.21$ & $4.35 \pm 0.45$ \\
\hline CAVPPRKA & $1 \pm 0.18$ & $1.02 \pm 0.41$ & $0.87 \pm 0.23$ \\
\hline ATF2/ATF3/ATF4 & $1 \pm 0.07$ & $2.24 \pm 0.15$ & $4.64 \pm 0.44$ \\
\hline
\end{tabular}

C

\begin{tabular}{c|c|c|c|c|c|c|c|}
\multirow{2}{*}{ Pathway } & Transcription & \multicolumn{4}{|c|}{ MG63 } & \multicolumn{3}{|c|}{ MG63.2 } \\
\cline { 3 - 7 } & Factor & NC & 3PA & 5PA & NC & 3PM & 5PM \\
\hline TGF & SMAD2/3/4 & $1 \pm 0.15$ & $0.79 \pm 0.02$ & $0.84 \pm 0.14$ & $1 \pm 0.02$ & $2.95 \pm 0.19$ & $1.56 \pm 0.11$ \\
\hline NFkB & NFKB & $1 \pm 0.03$ & $0.71 \pm 0.15$ & $0.57 \pm 0.10$ & $1 \pm 0.08$ & $1.38 \pm 0.08$ & $1.34 \pm 0.02$ \\
\hline Myc/Max & Myc/Max & $1 \pm 0.03$ & $0.59 \pm 0.02$ & $0.94 \pm 0.02$ & $1 \pm 0.16$ & $1.92 \pm 0.04$ & $1.49 \pm 0.08$ \\
\hline cAMP/PKA & CREB & $1 \pm 0.10$ & $0.70 \pm 0.07$ & $0.73 \pm 0.04$ & $1 \pm 0.07$ & $1.25 \pm 0.13$ & $3.21 \pm 0.09$ \\
\hline Hypoxia & HIF1A & $1 \pm 0.04$ & $0.76 \pm 0.25$ & $0.94 \pm 0.26$ & $1 \pm 0.12$ & $2.36 \pm 0.01$ & $0.70 \pm 0.09$ \\
\hline MAPK/JNK & FOS/JUN & $1 \pm 0.05$ & $0.95 \pm 0.12$ & $0.50 \pm 0.09$ & $1 \pm 0.05$ & $0.49 \pm 0.12$ & $0.87 \pm 0.04$ \\
\hline ATF2/ATF3/ATF4 & ATF2/ATF3/ATF4 & $1 \pm 0.25$ & $2.85 \pm 0.06$ & $1.42 \pm 0.05$ & $1 \pm 0.21$ & $0.75 \pm 0.05$ & $0.58 \pm 0.14$ \\
\hline
\end{tabular}

Fig. 6 The signaling pathways regulated by miR-193a-3p, miR193a-5p and their downstream genes. The experimental scheme (a). The relative activities (mean $\pm \mathrm{SD}$ ) of seven pathways that differed by more than two-fold between MG63 and MG63.2 cells (b). The relative pathway activities in the miR-193a-3p and miR-193a-5p

Rab27B and SRR are involved in the miR-193a-3pand miR-193a-5p-induced suppression of osteosarcoma cell migration and invasion

To clarify the biological roles of Rab27B and SRR in osteosarcoma cells, we knocked down endogenous Rab27B and SRR expression using specific small interfering RNAs (siRNAs) in MG63.2 cells. As shown in Fig. 5a, b, Rab27B and SRR mRNA and protein levels were significantly mimic (3PM/5PM)-transfected versus NC-transfected MG63.2 cells as well as miR-193a-3p and miR-193a-5p antagomiR (3PA/5PA)transfected versus NC-transfected MG63 cells (c). The relative pathway activities in the si-Rab27B- and si-SRR-transfected versus NC-transfected MG63.2 cells (d)

reduced by the siRNAs. In addition, wound-healing and invasion assays showed that metastatic MG63.2 cell migration and invasion were also markedly inhibited by the transfection of both si-Rab27B and si-SRR (Fig. 5c, d).

We next determined if Rab27B and SRR dysregulation was involved in the miR-193a-3p- and miR-193a-5p-induced migration and invasion of osteosarcoma cells. The 3PA and si-Rab27B constructs were transfected separately or together into MG63 cells. As shown by western blot 


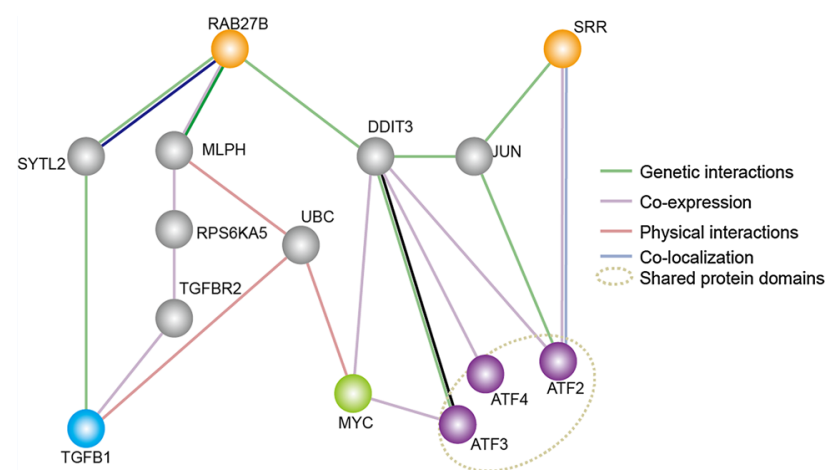

Fig. 7 A simplified interaction map was analyzed between the Rab27B and SRR target genes and the TGF $\beta$, Myc/Max and ATF2/ ATF3/ATF4 pathways using GeneMANIA (http://genemania.org/). Orange nodes represent the target genes. Gray nodes represent the genes that were related to target genes according to the GeneMANIA method. Blue, green and violet nodes represent the master transcription factor genes for the TGF $\beta$, Myc/Max and ATF2/ATF3/ATF4 pathways, respectively. The web-based interface searches yielded a large set of functional association data to return related genes based on available genomic and proteomic data. The association data include protein, DNA and genetic interactions as well as pathway data, gene expression data, protein expression data, phenotypic screens and shared protein domains. (Color figure online)

analysis in Fig. 5e, f, Rab27B expression was drastically increased by the transfection of 3PA in MG63 cells, whereas the co-transfection of both 3PA and si-Rab27B rescued Rab27B expression as well as the migration and invasion abilities of the cells (Fig. 5e, g, h). Similar results were also found with SRR in MG63 cells transfected with 5PA and/or si-SRR (Fig. 5f-h).

Furthermore, GFP-Rab27B and GFP-SRR proteins were over-expressed in MG63 cells. The ectopic expression of either Rab27B or SRR promoted MG63 cell migration and invasion (Fig. 5i-1).

Taken together, these results suggested that Rab27B and SRR are direct and functional targets of miR-193a-3p and miR-193a-5p, respectively, and that they are involved in the miRNA-induced suppression of osteosarcoma cell migration and invasion.

\section{MiR-193a-3p and miR-193a-5p regulate the TGF $\beta$, Myc/Max and ATF2/ATF3/ATF4 signaling pathways involved in osteosarcoma metastasis}

To gain further mechanistic insights into the roles of these miRNAs, we used the Cignal reporter finder assay (Fig. 6a) to compare the activities of 17 signaling pathways in MG63.2 versus MG63 cells. The MAPK/JNK and ATF2/ ATF3/ATF4 pathway activities were 2-fold and 12-fold higher, respectively, in MG63.2 cells than inMG63 cells (Fig. 6b). In contrast, the TGF $\beta, \mathrm{NF}-\kappa \mathrm{B}, \mathrm{Myc} / \mathrm{Max}, \mathrm{cAMP} /$ PKA and hypoxia pathway activities were significantly lower in MG63.2 cells than in MG63 cells (Fig. 6b). These seven differentially regulated pathways might play a significant role in osteosarcoma metastasis. Therefore, we compared the activities of these pathways in mimic-transfected MG63.2 cells and antagomiR-transfected MG63 cells against the control cells. The TGF $\beta$, NF- $\kappa B$, Myc/ Max, and cAMP/PKA pathways were down-regulated in the 3PA- or 5PA-transfected MG63 cells but up-regulated in the 3PM- or 5PM-transfected MG63.2 cells. The reverse effect was observed with the ATF2/ATF3/ATF4 pathway, which was activated in the 3PA- or 5PA-transfected MG63 cells and repressed in the 3PM- or 5PM-transfected MG63.2 cells (Fig. 6c). Furthermore, we compared the pathway activities inRab27B and SRR siRNA-transfected versus mock siRNA-transfected MG63.2 cells. Although no or marginal effects on the NF- $\kappa \mathrm{B}$ and cAMP/PKA pathway activities were observed, the TGF $\beta$, Myc/Max and ATF2/ATF3/ATF4 pathways were activated in either Rab27B or SRR siRNA-transfected MG63.2 cells (Fig. 6d). Notably, the Myc/Max pathway responded most drastically to Rab27B siRNA, whereas the ATF2/ATF3/ ATF4 pathway was up-regulated to the greatest extent by SRR siRNA. Therefore, the role of Rab27B/miR-193a-3p is mainly accomplished via its effect on the Myc/Max pathway, whereas the role of SRR/miR-193a-5p is mainly accomplished via the ATF2/ATF3/ATF4 pathway.

We then searched for interactions between the two target genes and the following master transcription factor genes of three signaling pathways (Fig. 7) using GeneMANIA (http://genemania.org/) [34]: TGFB1 for the TGF $\beta$ pathway, MYC for the Myc/Max pathway, and ATF2/ATF3/ ATF4 for the ATF2/ATF3/ATF4 pathway. Both RAB27B and SRR genetically interacted with ATF2/ATF3/ATF4 via DDIT3 and JUN, respectively. In contrast, the interaction of RAB27B and SRR with MYC was directed by the coexpression of DDIT3 and MYC. The interaction of RAB27B and TGFB1 occurred via three genes as follows: SYTL2, MLPH and UBC. Despite the lack of direct interaction between SRR and TGFB1, SRR may affect the TGF $\beta$ pathway through agenetic interaction with RAB27B. All of these results explain why RAB27B and SRR, the target genes of miR-193a-3p and miR-193a-5p, respectively, regulate the three afore mentioned signaling pathways.

\section{Discussion}

Aberrant miR-193a expression has been reported in many types of cancer, including colorectal cancer [33], NSCLC [24], epithelial ovarian cancer [35], myeloid leukemia [36] and Wilms' tumors [37]. In addition, according to previous studies, miR-193a-3p is also involved in cancer drug resistance through the repression of different target genes 
$[26,38]$. Here, we revealed for the first time that miR$193 a-3 p$ and miR-193a-5p are also involved in the suppression of osteosarcoma metastasis through two newly identified target genes, namely Rab27B and SRR.

MicroRNAs execute their biological function by repressing multiple genes at both the mRNA stability and translational levels. MiR-193a-3p and miR-193a-5p have been reported to target different genes that govern various types of cancer [27, 39]. In the present study, we showed that miR-193a-3p and miR-193a-5p were down-regulated in highly metastatic MG63.2 cells. The down-regulation of miR-193a-3p and miR-193a-5p correlated with the hypermethylated state of their promoter and enhancer regions in MG63.2 cells (Figs. 1, 2). Previous studies have indicated that DNA methylation is the best characterized epigenetic mechanism, and it is regarded as a promising molecular indicator for the existence and/or the prognostic state of cancer $[40,41]$. We next investigated target gene expression in highly metastatic MG63.2 cells compared with that in weakly metastatic MG63 cells. As expected, Rab27B and SRR mRNA and protein expression levels were increased in MG63.2 cells compared to MG63 cells (Fig. 4b-d). Moreover, increased Rab27B and SRR expression was correlated with decreased miR-193a-3p and miR-193a-5p expression (Fig. 4e-g). Hence, miR-193a-3p and miR-193a-5p negatively regulate osteosarcoma metastasis by targeting Rab27B and SRR, respectively.

Rab27B is a member of the RabGTPases, which constitute the largest family of small GTPases and play a vital role in endocytosis and exocytosis vesicle-trafficking control [42-45]. In addition, Rab27B proteins have been reported to be positively correlated with invasion and metastasis in colorectal cancer [46], hepatocellular carcinoma [47] and breast cancer [48-50], and these proteins mediate exosome secretion of tumor-related miRNAs and proteins such asproteases [51]. In agreement with these studies, our data suggested that Rab27B might facilitate the invasive/metastatic phenotypes of osteosarcoma and might thus represent a novel marker for clinical diagnosis and prognosis. SRR, the other identified gene, encodes a protein that catalyzes the synthesis of D-serine from L-serine. D-serine is a key co-agonist with glutamate at $N$-methyl-Daspartate (NMDA) receptors. Although no direct evidence has indicated a relationship between SRR and metastasis, NMDA receptors are associated with cell migration [52] and metastasis in oral squamous cell carcinoma [53]. Our work suggested for the first time that miR-193a-3p-regulated Rab27B and miR-193a-5p-regulated SRR contribute to the invasion and metastasis of osteosarcoma. However, the detailed mechanism awaits further investigation.

MiR-193a-3p and miR-193a-5p, two mature products from an identical precursor RNA, have previously been reported to suppress the metastasis of human NSCLC by down-regulating the ERBB4/PIK3R3/mTOR/S6K2 signaling pathway [28]. Additionally, miR-193a-5p depletion has been characterized as a marker of metastasis and poor prognosis in colorectal cancer [54]. Our present study showed for the first time that miR-193a-3p and miR-193a$5 p$ can suppress human osteosarcoma cell metastasis by suppressing two novel targets, Rab27B and SRR, respectively. Moreover, knocking down SRR inhibited the TGF$\beta, N F-\kappa B$, Myc/Max and ATF2/3/4 pathway activities, whereas knocking down Rab27B repressed the same three pathways, except for the NF- $\kappa B$ pathway, partially mimicking the effects of miR-193a-3p- and miR-193a-5pmimic transfection. The TGF- $\beta$ and Myc/Max pathways are well known for their positive impact on cancer metastasis $[55,56]$. ATF4 hasbeen reported to promote esophageal squamous cell carcinoma invasion and metastasis [57], whereas the effect of ATF3 on metastasis in different types of cancer remains controversial [58-61]. By inhibiting these pathways, Rab27B and SRR could be effective and functional downstream targets of miR-193a$3 p$ and miR-193a-5p in the mechanism of cancer metastasis. However, more details of this process and whether there is a synergistic effect between miR-193a-3p and miR193a-5p as well as their targets requires further investigation.

In this study, we demonstrated that miR-193a-3p and miR-193a-5p suppress the metastasis of human osteosarcoma cells by repressing Rab27B and SRR expression, through suppressing the TGF $\beta$, Myc/Max and ATF2/ ATF3/ATF4 signaling pathways. This study also provided a new set of genes that may be useful biomarkers for the diagnosis of osteosarcoma.

Acknowledgments This work was supported by the National Natural Science Foundation of China (81372868 Granted to SB) and the Natural Science Foundation of Anhui Province (1408085MH204 Granted to SB). We thank Dr. Luu from the University of Chicago for providing the MG63.2 cell line.

Author contributions Conception and design: YGP and SBC; Acquisition of data: YGP, FFZ, WJC, XHM, and YPL; Analysis and interpretation of data: YGP and FFZ; Writing, reviewing, and/or revising the manuscript: YGP and SBC. All authors read and approved the final manuscript.

\section{Compliance with ethical standards}

Conflict of interest The authors declare that they have no competing interests.

Open Access This article is distributed under the terms of the Creative Commons Attribution 4.0 International License (http://crea tivecommons.org/licenses/by/4.0/), which permits unrestricted use, distribution, and reproduction in any medium, provided you give appropriate credit to the original author(s) and the source, provide a link to the Creative Commons license, and indicate if changes were made. 


\section{References}

1. Botter SM, Neri D, Fuchs B (2014) Recent advances in osteosarcoma. Curr Opin Pharmacol 16:15-23

2. Yang J, Zhang W (2013) New molecular insights into osteosarcoma targeted therapy. Curr Opin Oncol 25(4):398-406

3. Kaste SC et al (1999) Metastases detected at the time of diagnosis of primary pediatric extremity osteosarcoma at diagnosis: imaging features. Cancer 86(8):1602-1608

4. Letson GD, Muro-Cacho CA (2001) Genetic and molecular abnormalities in tumors of the bone and soft tissues. Cancer Control 8(3):239-251

5. Fuchs B, Pritchard DJ (2002) Etiology of osteosarcoma. Clin Orthop Relat Res 397:40-52

6. Ragland BD et al (2002) Cytogenetics and molecular biology of osteosarcoma. Lab Investig 82(4):365-373

7. Su Y et al (2009) Establishment and characterization of a new highly metastatic human osteosarcoma cell line. Clin Exp Metastasis 26(7):599-610

8. Su Y et al (2011) Insulin-like growth factor binding protein 5 suppresses tumor growth and metastasis of human osteosarcoma. Oncogene 30(37):3907-3917

9. Sun K, Lai EC (2013) Adult-specific functions of animal microRNAs. Nat Rev Genet 14(8):535-548

10. Ameres SL, Zamore PD (2013) Diversifying microRNA sequence and function. Nat Rev Mol Cell Biol 14(8):475-488

11. Pritchard CC, Cheng HH, Tewari M (2012) MicroRNA profiling: approaches and considerations. Nat Rev Genet 13(5):358-369

12. Ambros V (2004) The functions of animal microRNAs. Nature 431(7006):350-355

13. Bartel DP (2004) MicroRNAs: genomics, biogenesis, mechanism, and function. Cell 116(2):281-297

14. Bartel DP (2009) MicroRNAs: target recognition and regulatory functions. Cell 136(2):215-233

15. Lewis BP, Burge CB, Bartel DP (2005) Conserved seed pairing, often flanked by adenosines, indicates that thousands of human genes are microRNA targets. Cell 120(1):15-20

16. Feng B et al (2012) Colorectal cancer migration and invasion initiated by microRNA-106a. PLoS One 7(8):e43452

17. Goldberger $N$ et al (2013) Inherited variation in miR-290 expression suppresses breast cancer progression by targeting the metastasis susceptibility gene Arid4b. Cancer Res 73(8):26712681

18. Yang H et al (2013) MicroRNA-140-5p suppresses tumor growth and metastasis by targeting transforming growth factor beta receptor 1 and fibroblast growth factor 9 in hepatocellular carcinoma. Hepatology 58(1):205-217

19. Zheng B et al (2011) MicroRNA-148a suppresses tumor cell invasion and metastasis by downregulating ROCK1 in gastric cancer. Clin Cancer Res 17(24):7574-7583

20. Chen L et al (2013) miR-16 inhibits cell proliferation by targeting IGF1R and the Raf1-MEK1/2-ERK1/2 pathway in osteosarcoma. FEBS Lett 587(9):1366-1372

21. Cao ZQ, Shen Z, Huang WY (2013) MicroRNA-802 promotes osteosarcoma cell proliferation by targeting p27. Asian Pac J Cancer Prev 14(12):7081-7084

22. Luo XJ et al (2014) MicroRNA-212 inhibits osteosarcoma cells proliferation and invasion by down-regulation of Sox4. Cell Physiol Biochem 34:2180-2188

23. Almeida MI et al (2012) Strand-specific miR-28-5p and miR-28$3 p$ have distinct effects in colorectal cancer cells. Gastroenterology 142(4):886-896

24. Heller $\mathrm{G}$ et al (2012) Genome-wide miRNA expression profiling identifies miR-9-3 and miR-193a as targets for DNA methylation in non-small cell lung cancers. Clin Cancer Res 18(6):1619-1629
25. Avci CB et al (2013) Therapeutic potential of an anti-diabetic drug, metformin: alteration of miRNA expression in prostate cancer cells. Asian Pac J Cancer Prev 14(2):765-768

26. Deng $\mathrm{H}$ et al (2014) miR-193a-3p regulates the multi-drug resistance of bladder cancer by targeting the LOXL4 gene and the oxidative stress pathway. Mol Cancer 13:234

27. Yang $Y$ et al (2013) A novel miR-193a-5p-YY1-APC regulatory axis in human endometrioid endometrial adenocarcinoma. Oncogene 32(29):3432-3442

28. Yu T et al (2015) MicroRNA-193a-3p and -5p suppress the metastasis of human non-small-cell lung cancer by downregulating the ERBB4/PIK3R3/mTOR/S6K2 signaling pathway. Oncogene 34(4):413-423

29. Shiraishi M, Hayatsu H (2004) High-speed conversion of cytosine to uracil in bisulfite genomic sequencing analysis of DNA methylation. DNA Res 11(6):409-415

30. Hayatsu H, Negishi K, Shiraishi M (2004) Accelerated bisulfitedeamination of cytosine in the genomic sequencing procedure for DNA methylation analysis. Nucleic Acids Symp Ser (Oxf) 48:261-262

31. Tahiri A et al (2014) Deregulation of cancer-related miRNAs is a common event in both benign and malignant human breast tumors. Carcinogenesis 35(1):76-85

32. Chen D et al (2013) MicroRNA deregulations in head and neck squamous cell carcinomas. J Oral Maxillofac Res 4(1):e2

33. Yong FL, Law CW, Wang CW (2013) Potentiality of a triple microRNA classifier: miR-193a-3p, miR-23a and miR-338-5p for early detection of colorectal cancer. BMC Cancer 13:280

34. Montojo J et al (2014) GeneMANIA: fast gene network construction and function prediction for Cytoscape. F1000Research 3:153

35. Wang $\mathbf{J}$ et al (2013) Demethylation of miR-9-3 and miR-193a genes suppresses proliferation and promotes apoptosis in nonsmall cell lung cancer cell lines. Cell Physiol Biochem 32(6): 1707-1719

36. Gao XN et al (2011) MicroRNA-193a represses c-kit expression and functions as a methylation-silenced tumor suppressor in acute myeloid leukemia. Oncogene 30(31):3416-3428

37. Watson JA et al (2013) miRNA profiles as a predictor of chemoresponsiveness in Wilms' tumor blastema. PLoS One $8(1): \mathrm{e} 53417$

38. Ma K et al (2012) DNA methylation-regulated miR-193a-3p dictates resistance of hepatocellular carcinoma to 5-fluorouracil via repression of SRSF2 expression. J Biol Chem 287(8):5639-5649

39. Lv L et al (2014) The DNA methylation-regulated miR-193a-3p dictates the multi-chemoresistance of bladder cancer via repression of SRSF2/PLAU/HIC2 expression. Cell Death Dis 5:e1402

40. Bonnette MD et al (2009) dcDegenerate oligonucleotide primedPCR for multilocus, genome-wide analysis from limited quantities of DNA. Diagn Mol Pathol 18(3):165-175

41. Lin Q et al (2009) RASSF1A, APC, ESR1, ABCB1 and HOXC9, but not p16INK4A, DAPK1, PTEN and MT1G genes were frequently methylated in the stage I non-small cell lung cancer in China. J Cancer Res Clin Oncol 135(12):1675-1684

42. Fukuda M (2008) Regulation of secretory vesicle traffic by Rab small GTPases. Cell Mol Life Sci 65(18):2801-2813

43. Pereira-Leal JB, Seabra MC (2001) Evolution of the Rab family of small GTP-binding proteins. J Mol Biol 313(4):889-901

44. Zerial M, McBride H (2001) Rab proteins as membrane organizers. Nat Rev Mol Cell Biol 2(2):107-117

45. Pfeffer SR (2005) Structural clues to Rab GTPase functional diversity. J Biol Chem 280(16):15485-15488

46. Bao J et al (2014) Rab27b is a potential predictor for metastasis and prognosis in colorectal cancer. Gastroenterol Res Pract 2014:913106 
47. Dong WW et al (2012) Differential expression of Rab27A/B correlates with clinical outcome in hepatocellular carcinoma. World J Gastroenterol 18(15):1806-1813

48. Zhang JX et al (2012) Overexpression of the secretory small GTPase Rab27B in human breast cancer correlates closely with lymph node metastasis and predicts poor prognosis. J Transl Med 10:242

49. Hendrix A et al (2010) Effect of the secretory small GTPase Rab27B on breast cancer growth, invasion, and metastasis. J Natl Cancer Inst 102(12):866-880

50. Hendrix A et al (2013) Vacuolar H+ ATPase expression and activity is required for Rab27B-dependent invasive growth and metastasis of breast cancer. Int J Cancer 133(4):843-854

51. Ostenfeld MS et al (2014) Cellular disposal of miR23b by RAB27-dependent exosome release is linked to acquisition of metastatic properties. Cancer Res 74(20):5758-5771

52. Xiao L et al (2013) NMDA receptor couples Rac1-GEF Tiam1 to direct oligodendrocyte precursor cell migration. Glia 61(12): 2078-2099

53. Choi SW et al (2004) The expression of NMDA receptor 1 is associated with clinicopathological parameters and prognosis in the oral squamous cell carcinoma. J Oral Pathol Med 33(9):533-537

54. Zhang P et al (2014) Downregulation of miR-193a-5p correlates with lymph node metastasis and poor prognosis in colorectal cancer. World J Gastroenterol 20(34):12241-12248
55. Zhang Q, Yu N, Lee C (2014) Vicious cycle of TGF-beta signaling in tumor progression and metastasis. Am J Clin Exp Urol 2(2):149-155

56. Wolfer A, Ramaswamy S (2011) MYC and metastasis. Cancer Res 71(6):2034-2037

57. Zhu H et al (2014) Activating transcription factor 4 promotes esophageal squamous cell carcinoma invasion and metastasis in mice and is associated with poor prognosis in human patients. PLoS One 9(7):e103882

58. Wu ZY et al (2014) Activating transcription factor 3 promotes colon cancer metastasis. Tumour Biol 35(8):8329-8334

59. Cao H, Yang ZX, Jiang GQ (2013) Expression and clinical significance of activating transcription factor 3 in human breast cancer. Iran J Basic Med Sci 16(11):1151-1154

60. Yuan X et al (2013) ATF3 suppresses metastasis of bladder cancer by regulating gelsolin-mediated remodeling of the actin cytoskeleton. Cancer Res 73(12):3625-3637

61. Jan YH et al (2012) Adenylate kinase-4 is a marker of poor clinical outcomes that promotes metastasis of lung cancer by downregulating the transcription factor ATF3. Cancer Res 72(19):5119-5129 